\title{
Bariatric surgery: blessing or sometimes curse for the liver?
}

\author{
Dimitris Kehagias ${ }^{1}$, Francesk Mulita ${ }^{1}$, Nikolas Drakos ${ }^{1}$, Fotios Seretis ${ }^{1}$, Elias Liolis ${ }^{2}$, Ioannis Kehagias ${ }^{1}$ \\ 1'Department of Surgery, Division of Bariatric and Metabolic Surgery, General University Hospital of Patras, Greece \\ ${ }^{2}$ Department of Internal Medicine, Division of Oncology, General University Hospital of Patras, Greece
}

\begin{abstract}
Introduction: Nowadays morbid obesity has become a worldwide health issue and the use of bariatric surgery undoubtedly results not only in weight reduction but also in the improvement of comorbidities. Although bariatric surgery is the optimal choice for metabolic syndrome resolution and hepatic function improvement, there is evidence that in rare cases it may lead to aggressive steatohepatitis, acute liver failure, fibrosis, and deterioration of the overall prognosis, without having fully understood the underlying pathophysiological mechanisms.

Case report: In this case report we present a 45 -year-old female patient with morbid obesity, body mass index 80, who underwent long-limb Roux en Y gastric bypass (LL-RYGB) and was admitted to the emergency department with jaundice and impaired liver function laboratory tests on postoperative day 90 . The examinations revealed elevated bilirubin and transaminases, with prolonged prothrombin time and low albumin levels. A liver biopsy was performed and showed active steatohepatitis. The hepatic values were gradually further impaired and the decision for surgery, in order to reverse the hepatic dysfunction, was taken. A gastrostomy in the bypassed stomach was performed and the activation of the closed biliopancreatic loop led to clinical improvement and amelioration of the prognosis.

Conclusions: Patients at high risk for hepatic failure after bariatric surgery should be better evaluated preoperatively and a tailor-made approach should be applied in order to avoid such a disastrous complication.
\end{abstract}

Key words: non-alcoholic fatty liver disease, bariatric surgery, gastrostomy.

\section{Introduction}

The number of patients with obesity is rapidly increasing, having reached 630 million worldwide, and these patients contend not only with the excess weight but also with serious comorbidities [1]. Bariatric surgery is the most effective tool available in the armamentarium and undoubtedly leads to weight loss and maintenance over time. According to the latest guidelines bariatric surgery is indicated in patients with body mass index (BMI) 40 and above or 35 and above with comorbidities, while sleeve gastrectomy (SG) and Roux en $\mathrm{Y}$ gastric bypass (RYGB) are the most common procedures performed [2, 3].

One of the most serious complications of morbid obesity is nonalcoholic fatty liver disease (NAFLD). Thus, parallel to the epidemic of morbid obesity, NAFLD is becoming the main liver disease with a frequency of $24-25 \%$ as well as the basic reason for liver transplantation [4-5]. It has a wide range of presentation, from simple steatosis to nonalcoholic steatohepatitis (NASH), fibrosis and hepatocellular carcinoma [6]. Over $80 \%$ of morbidly obese patients suffer from NAFLD,
$25-55 \%$ of them end up with NASH and $2-12 \%$ develop liver cirrhosis [7].

Various studies confirm that bariatric surgery improves, in the majority of patients, NAFLD and even signs of cirrhosis [8-9]. Unfortunately this is not the rule, and some studies, even from early 1970s, focused on the hepatic complications after malabsorptive techniques such as jejunoileal bypass (JIB), which was performed in a high rate at the beginning of bariatric surgery [10]. Decompensation of liver function and aggressive steatohepatitis is an uncommon complication after bariatric surgery and the underlying mechanisms are poorly understood [11].

Nowadays having abandoned such significant malabsorptive techniques as biliopancreatic diversion (BPD) and JIB, hepatic impairment is an even rarer complication. However, such cases continue to be reported in the literature, and understanding the risk factors and mechanisms is very important. Herein we present a rare case of a female patient with BMI 80, type 2 diabetes mellitus (T2DM) and arterial hypertension, who underwent LL-RYGB which resulted in hepatic impairment. 
Table 1. Laboratory findings of the patient at admission to the emergency department, before the gastrostomy, and 15, 30, and 60 days after the gastrostomy placement

\begin{tabular}{|c|c|c|c|c|c|c|}
\hline Laboratory findings & $\begin{array}{c}\text { Admission to emergency } \\
\text { department }\end{array}$ & $\begin{array}{l}\text { Before } \\
\text { gastrostomy }\end{array}$ & $\begin{array}{l}15 \text { days after } \\
\text { gastrostomy }\end{array}$ & $\begin{array}{l}30 \text { days after } \\
\text { gastrostomy }\end{array}$ & $\begin{array}{l}60 \text { days after } \\
\text { gastrostomy }\end{array}$ & $\begin{array}{l}\text { Normal } \\
\text { values }\end{array}$ \\
\hline Total bilirubin (mg/dl) & 3.34 & 22.7 & 6.16 & 2.09 & 1.86 & $0.1-1.3$ \\
\hline Conjugated bilirubin (mg/dl) & 2.58 & 12.7 & 5.28 & 1.71 & 1.22 & $<0.4$ \\
\hline Albumin (g/dl) & 2.7 & 2.7 & 2.7 & 3 & 3.2 & $3.5-5.5$ \\
\hline Total protein $(\mathrm{g} / \mathrm{dl})$ & 6.7 & 5.6 & 5.5 & 5.6 & 6.5 & $6-8.4$ \\
\hline $\operatorname{ALP}(U / L)$ & 210 & 128 & 138 & 190 & 207 & 34-104 \\
\hline GGT (U/L) & 439 & 157 & 134 & 176 & 406 & $10-50$ \\
\hline ALT (SGPT) & 54 & 29 & 57 & 38 & 20 & $5-40$ \\
\hline AST (SGOT) & 124 & 80 & 139 & 80 & 71 & $5-40$ \\
\hline $\mathrm{Hb}(\mathrm{g} / \mathrm{dl})$ & 14.2 & 11.5 & 10.1 & 8.3 & 12 & $11.8-17.8$ \\
\hline Cholesterol (mg/dl) & 353 & 250 & 131 & 119 & 160 & $<200$ \\
\hline Triglycerides (mg/dl) & 343 & 350 & 288 & 194 & 90 & $<150$ \\
\hline INR & 1.74 & 1.2 & 1.07 & 1.08 & 1.06 & 1 \\
\hline Urea (mg/dl) & 8 & 5 & 15 & 30 & 24 & $15-54$ \\
\hline $\mathrm{Cr}(\mathrm{mg} / \mathrm{dl})$ & 0.7 & 0.8 & 0.6 & 0.6 & 0.8 & $0.9-1.6$ \\
\hline
\end{tabular}

\section{Case report}

A female patient, 45 years old, with BMI 80, arterial hypertension, dyslipidemia, waist circumference $135 \mathrm{~cm}$, with medications for T2DM, underwent scheduled LL-RYGB (biliopancreatic limb $200 \mathrm{~cm}$, alimentary limb $150 \mathrm{~cm}$ ). On the fifth postoperative day leakage of the enteroenteric anastomosis was diagnosed, small bowel resection (approximately $100 \mathrm{~cm}$ ) with a new anastomosis was performed and she was discharged in good condition. 60 days after the surgery, the patient had an excess weight loss (EWL) of $38 \%$ but she gradually showed deterioration in the values of hepatic function and lipids (Table 1). Jaundice was presented and she was readmitted to the Surgery Department for support and better investigation of this impairment. First a computed tomography scan and blood cultures were obtained in order to exclude sepsis. The results were negative and the ultrasound did not show biliary obstruction but revealed serious liver steatosis and perihepatic fluid. Despite the nutritional support with special supplements, total bilirubin reached $20 \mathrm{mg} / \mathrm{dL}$ and signs of liver failure and clinical deterioration were noted. A liver biopsy was obtained and revealed steatohepatitis with bridging fibrosis (S3A3F3, steatosis activity fibrosis).

Ultrasound guided catheterization of the bypassed stomach was decided in order to place a gastrostomy tube for activating the blinded biliopancreatic limb and reverse the vicious cycle of hepatic impairment. Catheterization with ultrasound guidance did not succeed, so the patient had general anesthesia and a gastrostomy was placed, with the patient recovering well after the operation. Enteral feeding was started from the gastrostomy in the bypassed stomach and after 60 days the hepatic function improved, with better values and res- olution of jaundice. Now the patient is in the $6^{\text {th }}$ month postoperatively with EWL 61\%, no longer having diabetes or hypertension, and having nearly normalized hepatic values (Table 1). The gastrostomy tube was removed and the patient feeds orally. Hepatic impairment after bariatric surgery remains a rare complication and such cases should be recorded in order to evaluate the risk factors and better understand the underlying mechanisms.

\section{Discussion}

The most common complications of morbid obesity are cardiovascular disease, type 2 diabetes (T2DM), obstructive sleep apnea and dyslipidemia. The hepatic manifestations of morbid obesity are NAFLD and NASH. A high percentage of patients with obesity (70-90\%) are diagnosed with simple steatosis and $47 \%$ of them progress to steatohepatitis. When NAFLD coexists with T2DM and dyslipidemia, patients with obesity are prone to advanced steatohepatitis and fibrosis. Other risk factors for hepatic complications are high BMI, hyperlipidemia and active steatohepatitis [4]. The early diagnosis and staging of steatohepatitis are of high importance, not only for the choice of procedure but also for the correct follow-up of the patient, and this is achieved only with liver biopsy [11]. Single NAFLD is not an indication for bariatric surgery, although after the operation there is confirmed reduction of the steatosis and improvement of the histologic features in liver biopsy [12].

However, in the literature there are studies that describe an unfavorable course after bariatric surgery with hepatic impairment and aggravation of the steatohepatitis, with mechanisms not well understood [13]. The basic mechanism is rapid weight loss that provokes 


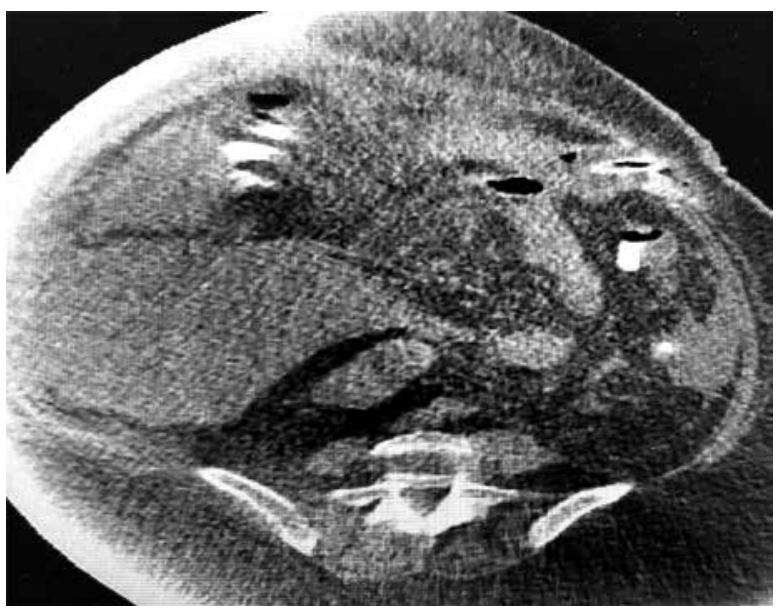

Fig. 1. Gastrostomy tube placed inside the bypassed stomach

massive accumulation of free fatty acids in the liver, and despite the rise of triglycerides in the liver, which is a defensive mechanism, the lipotoxicity leads to oxidative stress, aggressive steatohepatitis and fibrosis. This has been established as the first hit hypothesis. Other factors that contribute to this deterioration are bad nutrition status because of the malabsorption, with low protein intake and change in gut microbiota because of the closed biliopancreatic loop, leading to small intestine bacterial overgrowth (SIBO). It is also believed that the reabsorption of bile salts, which may be toxic for the liver, and perhaps genetic predisposition are also responsible for the hepatic impairment. So the existing evidence concludes in a multiple hit hypothesis, where multiple factors and mechanisms poorly understood lead to aggressive steatohepatitis and liver failure in high risk patients after bariatric surgery [14].

From a recent systematic review and meta-analysis of 23 studies, 59 patients with hepatic complications after bariatric surgery were recorded. In this meta-analysis a standard treatment approach is not established and various approaches are described, including liver transplantation, revision of the bariatric surgery, and surgical creation of gastrostomy along with nutritional support, but the mortality rate and the complications remain high [15].

In our case, a gastrostomy was created to the bypassed stomach (Fig. 1) in order to improve nutritional status, reverse SIBO and enhance the immune system, an approach which is suggested by some authors [16]. In high risk patients for development of aggressive steatohepatitis after bariatric surgery, more attention is needed to the rate of weight reduction and therefore to the choice of bariatric procedure. LL-RYGB, BPD and duodenal switch appear to correlate with a higher rate of hepatic complications due to the rapid weight loss they provoke [17]. The rate of BMI reduction cannot be predicted but studies show that a reduction $>1.6 \mathrm{~kg} /$ day leads to deterioration of steatohepatitis and fibrosis, especially in patients with high BMI [18].
A gold standard approach for obese patients at high risk for hepatic complications has not yet been established. Some authors suggest that performing SG followed by RYGB may have better outcomes in high risk patients because steatohepatitis is more easily self-limited [19].

\section{Conclusions}

Although bariatric surgery undoubtedly improves metabolic syndrome and resolves steatohepatitis in a high percentage of cases, hepatic impairment should be considered as a rare complication in high risk patients. Because of the paucity of cases recorded, the risk factors and the underlying pathophysiologic mechanisms are poorly understood [20].

Consequently, high risk patients should be carefully evaluated pre-operatively, with liver biopsy and staging of the liver function in order to select the most appropriate type of bariatric procedure and avoid this disastrous complication. Finally, intra-operative liver biopsy and fixation of the bypassed stomach to the abdominal wall for minimal invasive percutaneous gastrostomy in these high risk patients may also be considered.

\section{Disclosure}

The authors report no conflict of interest.

\section{References}

1. GBD 2015 Obesity Collaborators, Afshin A, Forouzanfar MH, et al. health effects of overweight and obesity in 195 countries over 25 years. N Engl J Med 2017; 377: 13-27.

2. Yumuk V, Tsigos $C$, Fried $M$, et al. European guidelines for obesity management in adults Obes Facts 2015; 8: 402-424.

3. Szeliga J, Wyleżoł M, Major P, et al. Metabolic and bariatric surgery chapter of the association of polish surgeons. bariatric and metabolic surgery care standards. Wideochir Inne Tech Maloinwazyjne 2020; 15 391-394.

4. Byrne CD, Targher G. NAFLD: a multisystem disease. J Hepatol 2015; 62: S47-S64.

5. Younossi ZM, Koenig AB, Abdelatif D, Fazel Y, Henry L, Wymer M. Global epidemiology of nonalcoholic fatty liver disease-meta-analytic assessment of prevalence, incidence, and outcomes. Hepatol 2016; 64: 73-84.

6. Pallayova M, Taheri S. Non-alcoholic fatty liver disease in obese adults: clinical aspects and current management strategies. Clin Obes 2014; 4: 243-253.

7. Clark JM. The epidemiology of nonalcoholic fatty liver disease in adults. J Clin Gastroenterol 2006; 40: S5-S10.

8. Kral JG, Thung SN, Biron S, et al. Effects of surgical treatment of the metabolic syndrome on liver fibrosis and cirrhosis. Surg 2004; 135: 48-58.

9. Hafeez S, Ahmed MH. Bariatric surgery as potential treatment for nonalcoholic fatty liver disease: a future treatment by choice or by chance? J Obes 2013; 2013: 839275.

10. Requarth JA, Burchard KW, Colacchio TA, et al. Long-term morbidity following jejunoileal bypass. The continuing potential need for surgical reversal. Arch Surg 1995; 130: 318-325.

11. Tsai JH, Ferrell LD, Tan V, Yeh MM, Sarkar M, Gill RM. Aggressive nonalcoholic steatohepatitis following rapid weight loss and/or malnutrition. Mod Pathol 2017; 30: 834-842. 
12. Younossi ZM, Stepanova M, Afendy M, et al. Changes in the prevalence of the most common causes of chronic liver diseases in the United States from 1988 to 2008. Clin Gastroenterol Hepatol 2011; 9: 524-530.

13. Liu X, Lazenby AJ, Clements RH, Jhala N, Abrams GA. Resolution of non alcoholic steatohepatits after gastric bypass surgery. Obes Surg 2007; 17: 486-492.

14. Cotler SJ, Vitello JM, Guzman G, Testa G, Benedetti E, Layden TJ. Hepatic decompensation after gastric bypass surgery for severe obesity. Dig Dis Sci 2004; 49: 1563-1568.

15. Vespasiani-Gentilucci U, Vorini F, Carotti S, et al. Hepatic complications of bariatric surgery: the reverse side of the coin. Acta Gastroenterol Belg 2017; 80: 505-513.

16. Keleidari B, Mahmoudieh M, Gorgi K, Sheikhbahaei E, Shahabi S. Hepatic failure after bariatric surgery: a systematic review. Hepat Mon 2019; 19): e86078.

17. Ralki M, Cassiman D, Van Dongen J, Ferrante M, Van Overbeke L. Liver failure after long-limb gastric bypass. Clin Res Hepatol Gastroenterol 2017; 41: e32-e37.

18. Shalhub S, Parsee A, Gallagher SF, et al. The importance of routine liver biopsy in diagnosing nonalcoholic steatohepatitis in bariatric patients. Obes Surg 2004; 14: 54-59.

19. Andersen T, Gluud C, Franzmann MB, Christoffersen P. Hepatic effects of dietary weight loss in morbidly obese subjects. J Hepatol 1991; 12 224-229.

20. Jan A, Narwaria M, Mahawar KK. A systematic review of bariatric surgery in patients with liver cirrhosis. Obes Surg 2015; 25: 1518-1526. 